\title{
Nonlinear EEG biomarker profiles for autism and absence epilepsy
}

William J. Bosl $1^{1,2^{*}}$ (i), Tobias Loddenkemper ${ }^{3,5}$ and Charles A. Nelson ${ }^{4,5}$

\author{
* Correspondence: \\ wjbosl@usfca.edu \\ ${ }^{1}$ Program in Health Informatics and \\ Clinical Psychology, University of \\ San Francisco, San Francisco, CA, \\ USA \\ ${ }^{2}$ Benioff Children's Hospital Oakland \\ Research Institute, Oakland, CA, USA \\ Full list of author information is \\ available at the end of the article
}

\begin{abstract}
Background: Although autism and epilepsy are considered to be different disorders, epileptiform EEG activity is common in people with autism even when overt seizures are not present. The relatively high comorbidity between autism and all epilepsy syndromes suggests the possibility of common underlying neurophysiological mechanisms. Although many different epilepsies may be comorbid with autism, absence epilepsy is a generalized epilepsy syndrome with seizures that appear as staring spells, with no motor signs and no focal lesions, making it more difficult to diagnose. Application of nonlinear methods for EEG signal analysis may enable characterization of brain activity that can help to delineate neurophysiological commonalities and differences between autism and epilepsy. Multiscale entropy and recurrence quantitative analysis (RQA) were computed from EEG signals derived from children with autism or absence epilepsy and compared with the goal of finding significant and potentially clinically useful biomarkers neurophysiological differences between these two childhood disorders.
\end{abstract}

Methods: Multiscale entropy and a multiscale version of RQA were computed from EEG data obtained from 92 children were collected in two different settings at Boston Children's Hospital. Short segments of alert resting state EEG were selected for analysis. A complexity index derived from entropy and RQA methods was computed from each of 19 standard EEG channels for all subjects using publicly available software. Statistical comparisons were made between the groups. Machine learning classifiers were also used to determine which derived features were most significantly different among the groups, and to determine classification specificity and sensitivity.

Results: Significant differences were found between absence, autism, and control groups in a number of different scalp locations and the values of complexity index. Autism values appeared to be intermediate between epilepsy and control in many locations, and differences between controls and absence patients were more widely distributed across scalp locations. Classification algorithms were able to distinguish absence epilepsy and autism cases from controls with high (>95\%) accuracy. Importantly, two independent control groups, although they were derived from different settings and with different equipment were statistically indistinguishable. (Continued on next page)

\section{Biomed Central}

(c) The Author(s). 2017 Open Access This article is distributed under the terms of the Creative Commons Attribution 4.0 International License (http://creativecommons.org/licenses/by/4.0/), which permits unrestricted use, distribution, and reproduction in any medium, provided you give appropriate credit to the original author(s) and the source, provide a link to the Creative Commons license, and indicate if changes were made. The Creative Commons Public Domain Dedication waiver (http://creativecommons.org/ publicdomain/zero/1.0/) applies to the data made available in this article, unless otherwise stated. 
(Continued from previous page)

Conclusions: Signficant neurophysiological differences were found between absence, autism, and control cases. In most scalp regions, autism values were intermediate between the control values and absence values, suggesting several future research studies. Nonlinear EEG signal analysis, together with classification methods, may provide complementary information to visual EEG analysis and clinical assessment in epilepsy and autism, and may provide useful information for research on pediatric neurodevelopmental and neurological disorders. Additional research may enable neurophysiological biomarker profiles to be derived from these techniques for clinical use.

Keywords: Epilepsy, Autism, EEG, Multiscale entropy, Nonlinear, Signals, Recurrence plot analysis, Machine learning

\section{Background}

Autism spectrum disorder (ASD, or simply 'autism') and epilepsy are common neurodevelopmental disorders that account for a large proportion of child and adult neurologic burden of disease [1], with co-morbidities as high as $30 \%$ or more [2-5]. Some researchers have suggested that the degree of clinical overlap between ASD and epilepsy arises from a common neurodevelopmental dysfunction [6]. The heterogeneous spectrum of symptoms displayed both disorders makes it particularly difficult to study commonalities and differences between these disorders. There is evidence of abnormal epileptiform abnormalities in children with ASD even in the absence of clinical seizures, but little is known about the clinical or therapeutic implications of this [7]. Genetic studies have found that variants of a single gene (SCN2A) could cause either gain of function and increased neuronal excitability resulting in seizures, or loss of function and decreased neuronal excitability leading to an outcome of ASD [8]. This suggests that similar mechanisms with variable outcomes may be involved in ASD and epilepsy.

ASD constitutes a heterogeneous developmental syndrome that is usually characterized by a triad of impairments that affect social interaction, communication skills, and a restricted range of interests and activities [9, 10]. ASD is not a single disorder, but rather a spectrum of various subtypes with different (largely unknown) causes and developmental trajectories. Current prevalence estimates are that 1 in 68 children now born the US will meet the criteria for an ASD diagnosis [11]. The incidence of autism globally is thought to be relatively uniform, although estimates vary greatly. A prevalence of 1 in 38 children in South Korea was reported [12]. In general, reliable data from low income countries is lacking. One study of global prevalence found no clear evidence of a change in prevalence for ASD between 1990 and 2010 [13]. Estimates of ASD prevalence as high as 1 in 38 children in South Korea have been reported [12]. Although prevalence of ASD is believed to be relatively uniform throughout the world, very little reliable data is available in low income countries [12], where the prevalence of epilepsy is estimated to be significantly higher than in high income countries [14].

Epilepsy has also been described as a heterogeneous neurological spectrum disorder [15] that is characterized by an enduring predisposition to generate epileptic seizures. Older definitions characterize epilepsy by at least two unprovoked seizures occurring at least $24 \mathrm{~h}$ apart, and by the neurobiological, cognitive, psychological, and social consequences of this condition [16]. A more recent definition accepted by the International League Against Epilepsy (ILAE) refines this definition to include reflex seizures that are 
provoked by, for example, flashing lights or other common environmental stimuli, and the possibility of outgrowing epilepsy [17]. Epilepsy in the US occurs in approximately $0.6 \%$ in children and $1 \%$ in adults for all epilepsies [18]. As previously mentioned, epilepsy is believed to be more common in low income regions, but data from these regions is lacking [14]. The paucity of reliable data on the prevalence of both ASD and epilepsy from the majority of the world's population suggests a critical need for further research. There is a continuing need for greater communication and research studies between the epilepsy and ASD communities to help shed light on the relationship between these disorders [7].

Although the relationship between ASD and epilepsy is not well understood, the high co-occurrence of these disorders suggests a common underlying neurodevelopmental pathology [6]. A link between epileptiform discharges and developmental disorders has been described in cases of 'subclinical' discharges without epilepsy [19, 20]. Even in the absence of overt seizures, reports of epileptiform activity in electroencephalograms (EEGs) in up to $60 \%$ of children with ASD have been reported [4, 21], and children with early onset seizures are at higher risk of developing ASD [22]. The incidence of epileptiform abnormalities is reported to correlate the severity of autistic symptoms [23]. Both epileptiform discharges and seizures are a manifestation of abnormal excessive synchronization of neurons, resulting in high amplitude discharges seen on EEG traces. A better understanding of the underlying cause of the shared susceptibility for ASD and epilepsy may create new opportunities for early intervention that can ameliorate or prevent symptoms from emerging [7]. Since electrophysiological abnormalities appear to be associated with both disorders, new approaches to EEG analysis are promising avenues for investigation.

Childhood absence epilepsy (AE, formerly known as petit mal epilepsy) is a subtype of generalized idiopathic epilepsy. The hallmark of an absence seizure is an abrupt loss of consciousness, usually without motor impairment that may last a few seconds to half a minute [24]. Because of the lack of overt seizures, the symptoms of AE are sometimes initially misdiagnosed as an attention deficit problem (ADHD) [25-27]. Absence seizures in children with ASD may not be detected because the seizure symptomology may be similar to behavioral symptoms of autism [7].

A specific seizure type is not known to be more common to ASD [7], but seizures with motor symptoms or focal lesions may be easier to detect. AE was chosen for this study because it is relatively common in childhood, lacks of identifiable lesions, seizure focus, and motor signs, which make it more difficult to easily distinguish from ASD.

Various measures of nonlinear dynamics have been computed from EEG time series in order to detect changes immediately prior to the onset of seizures or epileptiform discharges. Permutation entropy was found to change significantly up to $5 \mathrm{~s}$ before seizure onset in rat models of AE [28]. Kolmogorov entropy, Correlation Dimension [29], Relative Wavelet Energy [30] and Approximate Entropy [31] have all demonstrated some success for detecting pre-seizure onset periods, but could not distinguish healthy controls from epilepsy patients during seizure-free periods. Mixed results have been reported for automated seizure detection algorithms based on four different measures (principal eigenvalue, total power, Kolmogorov entropy, correlation dimension). The algorithms were found to be patient-age specific and no single algorithm performed well on all patients [32]. 
Application of EEG analysis to the detection of ASD is more recent, but has shown some success. Catarino et al. found significantly decreased multiscale entropy in ASDdiagnosed participants compared to controls [33]. Bosl et al., found the same trend in multiscale entropy in infants who were not old enough to exhibit the defining characteristics of ASD. The latter found that the greatest differences are observed between 9 and 12 months of age, as multiscale entropy shows an overall different developmental trajectory for infants at high-risk of developing ASD compared to low-risk infants [34]. Elridge et al. used Bayesian methods to perform a similar classification between ASD and typically developing children, from 6-10 years old. This study extracted robust features such as variance in time, entropy or sum of signed differences from the EEG signal and then used logistic regression and a native Bayes classifier to divide the two groups with a $79 \%$ accuracy [35].

A relatively recent approach to nonlinear system characterization, based on statistical analysis of recurrence plots, is called recurrence quantitative analysis or RQA [36-38]. RQA is an empirical approach to analyzing time series data and is in principle capable of characterizing all of the essential dynamics of a complex system and is useful for analyzing "real-world, noisy, high dimensional data" [39]. It has proven to be a powerful tool already in physics, geophysics, engineering and biology [36, 40]. Applications to neurology and neuroscience are in the early stages. In principle, RQA is capable of detecting significant state changes in a dynamical system [36, 37, 41], which suggests that it may be appropriate for detecting developmental changes in brain function that are associated with chronic neurological and mental dysfunction. Recurrence quantitative analysis has been used for early seizure detection by distinguishing ictal and inter-ictal entropy states [42-44] and recently for differentiating children with ASD from typically developing children [45].

We performed a quantitative analysis to evaluate the use of nonlinear features derived from multiscale entropy and RQA applied to EEG segments as biomarkers for AE and ASD. The goal of this study was to perform characterize and compare the electrophysiological dynamics of the brains of children with ASD and AE, using broad nonlinear analysis techniques, in order to contribute to understanding commonalities and differences between these disorders. A secondary goal was to introduce nonlinear signal analysis, feature selection, and machine learning as tools for discovery in neurophysiology research.

\section{Methods}

This study was performed at two different settings within Boston Children's Hospital $(\mathrm{BCH})$. Epilepsy patients and a control group were seen at a tertiary epilepsy center in the Division of Epilepsy and Clinical Neurophysiology, Department of Neurology, Boston Children's Hospital (BCH). A flowchart illustrated the data processing steps followed is shown in Fig. 1. Details for each step are described below.

Data were collected retrospectively from a database of patients who had undergone routine EEG at the tertiary epilepsy center in the Division of Epilepsy and Clinical Neurophysiology, Department of Neurology, Boston Children's Hospital (BCH). The Institutional Review Board at Boston Children's Hospital granted approval for the use of this data for this study. Given the retrospective nature of the clinical epilepsy data, the need to obtain individual informed consents was waived for these subjects. 


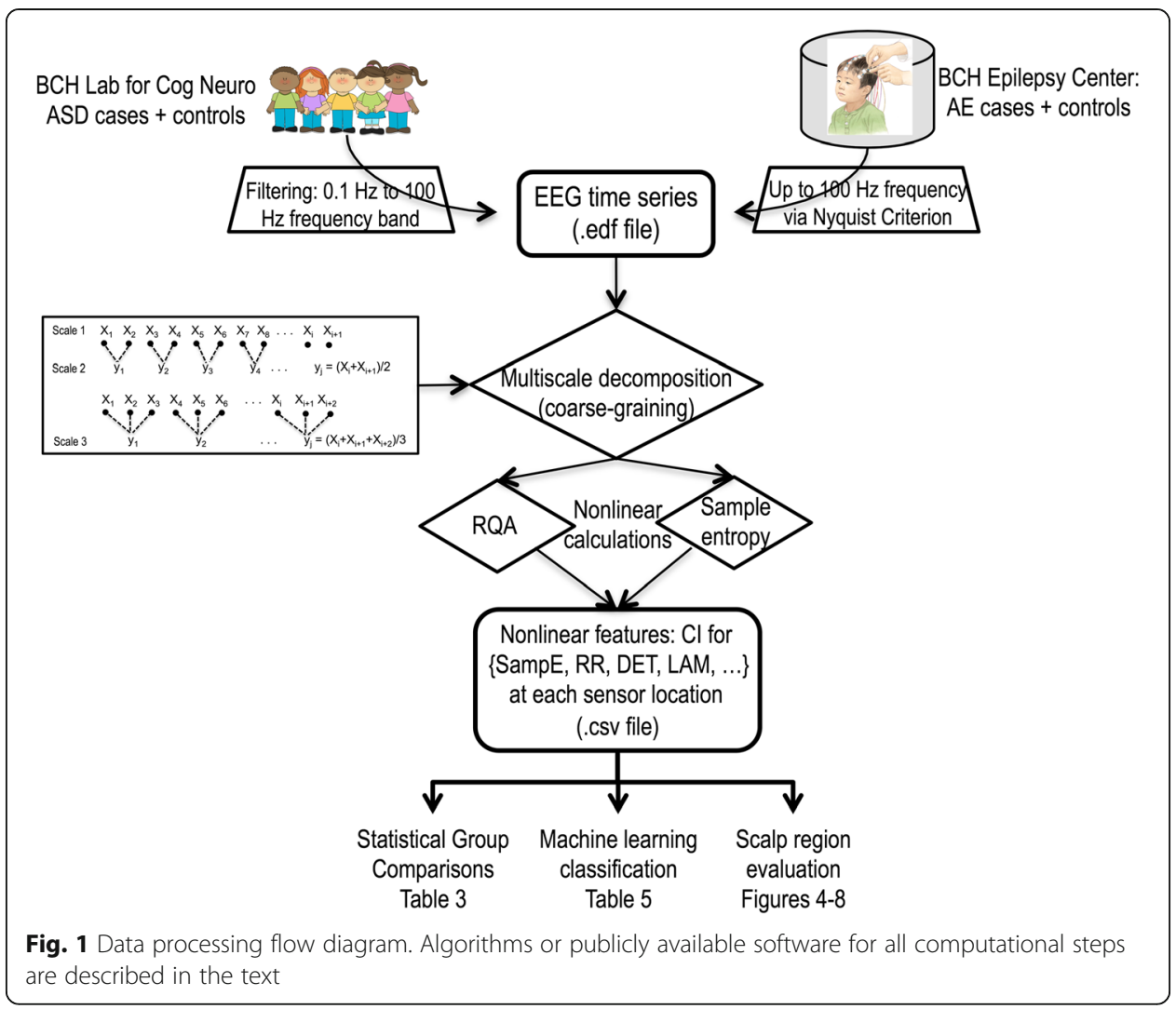

ASD patient data were acquired in the Laboratories for Cognitive Neuroscience (LCN) in the Developmental Medicine Division at BCH. Children with ASD and controls were recruited specifically for participation in this study. The study was approved by the Committee on Clinical Investigations at Boston Children's Hospital. Parental written informed consent was obtained after the experimental procedures had been fully explained.

The main demographic features of all subjects are summarized in Table 1. Twentyfour AE cases and 18 ASD cases are included, along with 49 controls from both settings: 24 controls from the epilepsy clinic and 23 controls from the Laboratories of Cognitive Neuroscience.

\section{BCH epilepsy clinic}

A database of patients undergoing routine electroencephalograms (EEG) performed at the $\mathrm{BCH}$ Epilepsy center was reviewed retrospectively and two populations of subjects were identified: patients with typical absence seizures and patients that underwent an

Table 1 Numbers and demographic features of the study population

\begin{tabular}{|c|c|c|c|c|}
\hline \multirow[t]{3}{*}{ Parameter } & \multicolumn{2}{|c|}{ BCH epilepsy clinic } & \multicolumn{2}{|c|}{ Lab Cog Neuro (LCN) } \\
\hline & $\overline{\mathrm{AE}}$ & Control 1 & $\overline{A S D}$ & Control 2 \\
\hline & $N=26$ & $N=24$ & $N=18$ & $N=23$ \\
\hline Mean age in years (std dev) & $8.6(1.7)$ & $7.74(4.3)$ & $8.8(1.9)$ & $8.6(1.4)$ \\
\hline Gender (male/female) & $13 / 13$ & $9 / 15$ & $16 / 2$ & $21 / 2$ \\
\hline
\end{tabular}


EEG for different reasons but were eventually determined to not have epilepsy (control group). All subjects selected from the Epilepsy Center met the following inclusion criteria: 1) normal neuropsychological development, and 2) no other EEG abnormalities than those consistent with generalized AE. Diagnosis was confirmed by documented seizures on EEG, and the diagnosis of typical absence seizures was confirmed after careful evaluation of the clinical and EEG features by at least one board-certified clinical neurophysiologist. EEGs were measured with a Natus Neuroworks XLTEK EMU 40 system, and with NeuroWorks software version 8.

The epilepsy control group met the following criteria: 1) at least one EEG study because of the clinical suspicion of seizures, 2) normal routine EEG study after visual inspection, 3) very low-risk of a diagnosis of epilepsy after a thorough electro-clinical evaluation. Causes for performing an EEG study in controls included daydreaming, syncope, night terrors or sleepwalking.

EEG data was sampled at $200 \mathrm{~Hz}$ for all Epilepsy Center subjects. Based on the Nyquist criterion, this implies that frequencies up to $100 \mathrm{~Hz}$ are included. From the $\mathrm{AE}$ cases, an experienced neurologist not directly involved in this study used visual review to select 30-s samples containing no spikes or evidence of epileptiform activity. Similarly, 30-s segments were selected from the control group after visual review. These samples contain 6000 points in each EEG sensor time series. A single segment from each subject was provided for analysis. The control group taken from the Epilepsy Center will be referred to as the 'control 1' group when necessary to distinguish is from the 'control 2' group. All EEG samples collected in the Epilepsy Center were from awake subjects and the segments chosen appeared normal to the neurophysiologist on visual analysis. For all subjects, segments of equal length were collected on 19 channels located according to the standard 10-20 system. We note that a single continuous segment, without visible epileptiform activity and without visual artifacts were selected from each subject. No other filtering was performed on the EEG signals.

\section{Laboratory of cognitive neuroscience autism study subjects}

The autism study group of participants included 18 children with an autism spectrum disorder (mean age $=9.0$ years; $\mathrm{SD}=1.7 ; 16$ males, 2 females) and 23 typically developing children (mean age $=8.6$ years; $\mathrm{SD}=1.4 ; 12$ males, 11 females). Participants were recruited from a list of families who had expressed interest in research participation. ASD diagnosis was confirmed by clinical diagnosis as reported by the parent and/or by meeting criteria on the Autism Diagnostic Observation Schedule (ADOS) in a research setting conducted within the past year.

All LCN electrophysiological recording was completed in an electrically and soundshielded testing room with low lighting. Children were seated on a chair approximately $60 \mathrm{~cm}$ in front of the experimental monitor. Continuous baseline EEG was recorded before children were presented with images or other stimuli. Only baseline data was used in this study.

Continuous EEG was recorded using a high density 128-channel Geodesic Sensor Net (Electrical Geodesics Inc., Eugene, OR). The electrical signal was amplified with a 0.1 to $100 \mathrm{~Hz}$ band-pass, digitized at $500 \mathrm{~Hz}$, and stored on a computer disk. The data were analyzed offline by using NetStation 4.4.1 analysis software (Electrical Geodesics 
Inc., Eugene OR). After using an artifact detection tool standard to the NetStation software package to exclude segments with eye saccades and blinks, the remaining segments were visually scanned by an experimenter blind to study group. For comparison to the subjects examined in the $\mathrm{BCH}$ Epilepsy Center, only 19 channels were selected from the sensor net, corresponding to the positions in the standard 10-20 montage [46]. As with all subjects, 6000 points were selected from each EEG time series.

\section{EEG analysis methods}

Each of the EEG samples was processed in an identical manner by the following steps. A single segment of 6000 points was selected from EEG data for each subject, as described above, from each of the respective settings. Average referencing was computed and used for all EEG time series. Data from all subjects were selected from the standard 10-20, 19-sensor montage locations.

Multiscale time series for scales 1 to 32 were derived from the original EEG signals using the coarse-graining procedure described in (Costa et al., 2005). This procedure is illustrated in Fig. 1. We note that for scales 1, 2, 4, 8, 16, and 32 the coarse-graining procedure is identical to the approximations derived with a Haar wavelet transform [47], though we did not use wavelets in this research. For this research, the coarsegraining algorithm was used. RQA values were computed on all scales 1 to 32 for each subject at each EEG electrode. The EEG times series for all subjects from both laboratories contain frequencies up to $100 \mathrm{~Hz}$. This was the Nyquist limit for the Epilepsy Center data, which had a sampling rate of 200 samples per second. The LCN data was filtered for frequencies of 0.1 to $100 \mathrm{~Hz}$.

RQA values were computed for all of the scales derived from each EEG channel using publicly available software [48]. For input to the algorithms, the embedding dimension (m) was 10 , the time delay was 2 , and the threshold for the recurrence plot (tau) was

30. For detailed discussions of how these values may be determined, see [41, 43, 44].

Multiscale sample entropy was also computed and included in this set of EEG signal features and is denoted by SampE as in [49]. Sample entropy and the entropy derived from RQA, denoted "L_entr", are expected to be similar quantitative measures, but derived from different algorithms. Thus, eight features or values were computed for each EEG sensor time series. Table 2 lists the nonlinear values computed for this study and provides a brief description of their meaning in a physical context.

The area under each multiscale curve, called a complexity index or CI [50], was computed by summing the value at each scale, then dividing by the total scale length. The area under the curve takes into account, to some extent, the starting point, slope, and convexity of the curve. For all comparisons in this paper, the CI for each measure (sample entropy, RR, DET, and so on) will refer to the area under the multiscale curve. Larger multiscale entropy has been reported for healthy physiological systems in general [51], but it is not clear what values should be expected for other nonlinear measures, such as those computed in recurrence quantitative analysis. Examples of the multiscale curves at Fp1 and Fp2 for the DET measure are shown in Fig. 2.

Nonlinear measures applied to physiologic data such as posturography data in children with autism, tend to be lower than in typical controls [52]. Similarly, the complexity index in patients with AE decreased across all EEG channels in a standard 
Table 2 Recurrence quantitative analysis variables and their interpretation

\begin{tabular}{|c|c|c|}
\hline RQA variable & Symbol & Description \\
\hline Recurrence rate & $\mathrm{RR}$ & $\begin{array}{l}\text { The probability that a system state recurs in a finite time. RR has been } \\
\text { found useful for detecting evoked response potentials (ERPs) using } \\
\text { single trials [30]. }\end{array}$ \\
\hline Determinism & DET & $\begin{array}{l}\text { DET comes from repeating patterns in the system and is an indication } \\
\text { of its predictability. Regular, deterministic signals, such as sine waves, will } \\
\text { have higher DET values, while uncorrelated time series, such as chaotic } \\
\text { processes and random numbers, will cause low DET. }\end{array}$ \\
\hline Laminarity & LAM & $\begin{array}{l}\text { Laminarity represents the frequency of occurrence of laminar states } \\
\text { in the system without describing the length of these laminar phases. } \\
\text { More frequent appearance of laminar states may relate to more frequent } \\
\text { "seeds" for synchronized dynamics [46], which may be related to } \\
\text { epileptiform spiking on an EEG trace. }\end{array}$ \\
\hline Max line length & L_max & $\begin{array}{l}\text { Lmax is related to the largest Lyapunov exponent of a chaotic signal, } \\
\text { which is a dynamic complexity measure that describes the divergence of } \\
\text { trajectories starting at nearby initial states, }[47] \text {. Lower values are typically } \\
\text { associated with pathological conditions }[43,48] \text {. }\end{array}$ \\
\hline $\begin{array}{l}\text { Entropy derived from } \\
\text { diagonal lines }\end{array}$ & L_entr & $\begin{array}{l}\text { This measure of entropy is derived from the diagonal lines of the } \\
\text { recurrence plot. It is related, but not identical to, other measures of } \\
\text { entropy, such as the sample entropy used in previous studies [37] }\end{array}$ \\
\hline Trapping time & $\pi$ & $\begin{array}{l}\text { Trapping time is an estimate of the time that a system will remain in a } \\
\text { given state - "trapped" state. Thus, lower } \Pi \text { values may be an indication } \\
\text { of more frequent transitions between dynamical states and less system } \\
\text { stability. }\end{array}$ \\
\hline
\end{tabular}

19 sensor montage during seizures [50], with greatest changes from pre-ictal to seizure states occurring in frontal and central regions. However, differences in complexity index between $\mathrm{AE}$ patients and controls during inter-ictal periods have not been reported.

For classification calculations, the features used were the complexity index for each feature described in Table 2, at each sensor location for a total of 152 features. A recursive feature elimination algorithm was used to rank the features and a standard Support Vector Machine (SVM) classifier was then used to classify each of the groups when compared to controls, as well as to compare the epilepsy and autism control groups (control 1 and control 2) to each other.

A recursive feature elimination algorithm was used to rank the features and a standard Support Vector Machine (SVM) classifier was then used to classify each of the groups when compared to controls, as well as to compare the control groups to each other.

Classification results shown in Table 4 were derived from 10-fold cross validation calculations. Feature ranking and selection were done using a recursive feature

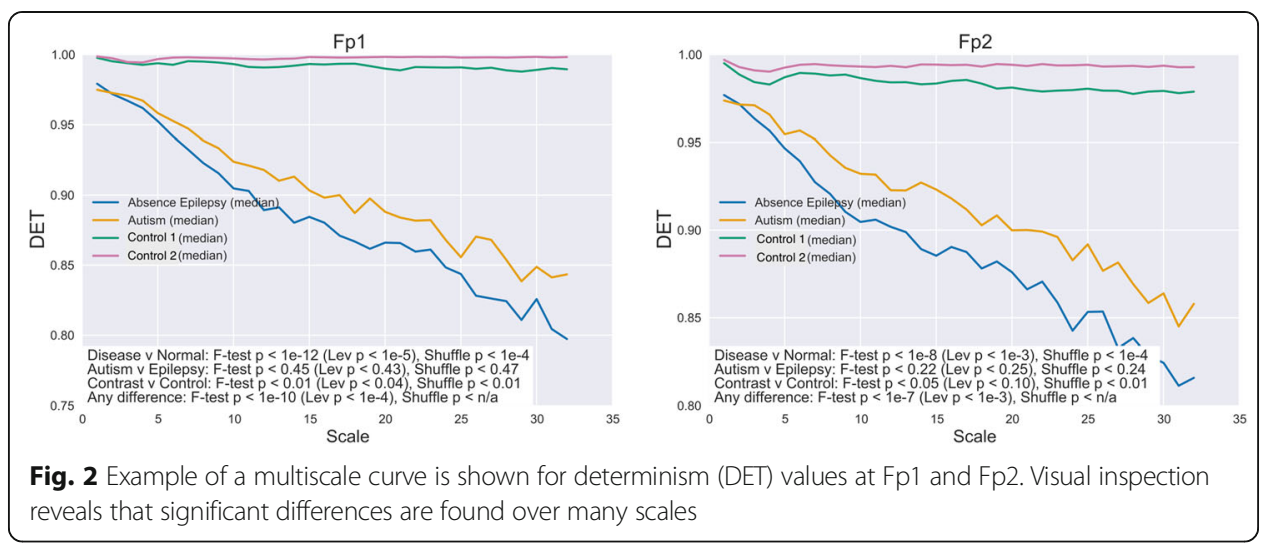


selection algorithm as implemented in the widely used, open source scikit-learn package (http://scikit-learn.org/stable/modules/cross_validation.html; [53]). Feature selection used the automatic selection call, pred $=\operatorname{rfecv}$.predict $(X)$, where $X$ is the matrix containing the features in columns and subjects in row. $\mathrm{Y}$ is an array of labels (ASD, AE, or control). Features consisted of the complexity index for each sensor and each of the features in Table 2. The code needed to reproduce these results is shown here:

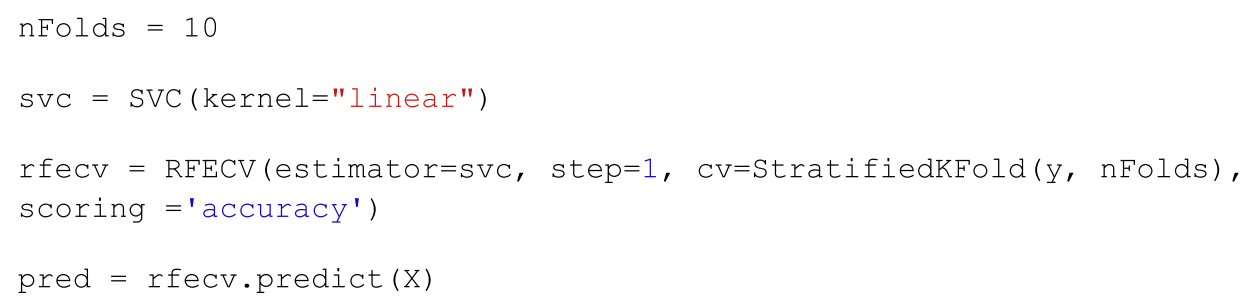

Recursive feature selection is used with a Support Vector Machine (SVC) classifier algorithm to recursively remove features that have low scores as SVC support vectors and build a model based on remaining features. Model accuracy is used to identify which features and combinations of features contribute most to classification accuracy.

The empirical $p$-values were computed using the method of permuting labels as described in [54]. The idea is to randomly assign the labels to subjects, then carry out the classification and compute accuracy. This process is repeated one hundred times. The number of times that the accuracy with permuted labels exceeds the accuracy with true labels is an estimate of the $p$-value. That is, it is an estimate of the probability that the accuracy determined with the true labels would have been computed if the null hypothesis (no group differences) were true.

\section{Results}

Multiscale RQA plots were computed for each of the 19 sensor locations in the standard 10-20 configuration. The multiscale time series were derived from the original EEG segments using a previously described averaging method [51]. Although methods for comparing curves based on mean value, slope and various shape parameters are possible, we chose in this study to use the mean value of the multiscale curve to represent the entire curve for simple comparisons between curves. Using the mean values only, group differences that met an overly strict Bonferroni-corrected criterion of $p<10^{-4}$, derived by dividing the commonly used significance cutoff of 0.05 by $19 * 8$ ( 19 sensors, 8 features) and rounding down to the nearest power of 10, are shaded in Figs. 4, 5, 6, 7 , 8. Multiscale curves were computed for the following RQA values: RR, DET, LAM, L_entr, L_mean, L_max and TT. In addition, the modified multiscale sample entropy (SampE) used in [49] was also computed. Collectively, these are referred to as 'signal features' or simply features hereafter. A single Complexity Index (CI) computed from the area under the multiscale curve has been introduced in the literature [55]. Previous studies have examined several RQA values (RR, DET, LAM) computed on a single scale from EEG time series. Determinism (DET) and Laminarity (LAM) were been found to be lower closer to the epileptic zone in patients with focal epilepsies, while recurrence rate (RR) and entropy were higher [56]. This suggests that similar differences in general 
might be found between $\mathrm{AE}$ cases and controls, since $\mathrm{AE}$ is not localized. Although we found some differences in the CI for SampE and all RQA values, when we use a strict significance criterion of $p<10^{-4}$, the most important nonlinear features were not RR, DET, and LAM, but the remaining features. The significance threshold was derived using a strict Bonferroni correction from the commonly used threshold of .05, dividing by the number of features (19 sensor locations times $8 \mathrm{CI}$ values) and rounding down to the nearest power of ten.

Table 3 summarizes the most significant group differences found when comparing group pairs at each sensor location and for each signal feature. The significance was computed using the two-tailed independent t-test found in the scipy.stats.ttest_ind package using default parameters. A surprising result was that the two control groups were indistinguishable. They did not have any significant differences at any sensor location for any signal feature. This is important because the EEG measurements were taken with different equipment, in two different settings: one a research laboratory, the other a busy tertiary epilepsy center. Furthermore, classification methods could not distinguish between control groups. For this reason, the control groups were combined for all other group comparisons.

\section{Absence versus controls}

The absence group differed significantly from the control at most sensor locations across the scalp for one or more signal features when comparing the mean value of the curve across all scales. The EEG segments were from inter-ictal cases and did not exhibit epileptiform activity. L_max and SampE were the most significantly different. Of the eight features, only two (L_mean and TT) did not meet the significance criterion of $p<10^{-4}$ at any location for any feature using mean values. If slope is considered, then group mean slopes for some sensor locations for L_mean and TT are significantly different.

\section{ASD versus controls}

The ASD group also differed significantly from the control groups. Similar signal features were significant as to the absence-control comparison, but scalp locations were not as widely distributed. Table 4 reveals that the most significant differences were at scalp locations in the orbitofrontal (Fp1, Fp2), left temporal (T7) and occipital (O1, O2) regions.

Table 3 The significance of group differences was computed using the two-tailed t-test for each sensor location and each signal feature

\begin{tabular}{lll}
\hline Classification & $\begin{array}{l}\text { Most significant sensor locations (Bonferroni } \\
\text { corrected significance level of } 10^{-4} \text { used) }\end{array}$ & Most significant nonlinear measures \\
\hline AE vs. controls & Frontal, occipital, temporal, parietal & SampE, L_entr, L_max, TT \\
& Fp1, Fp2, Fz, F8, T7, P7, Pz, P8 O1, O2 & \\
ASD vs. controls & Frontal, occipital, left temporal & SampE, L_max, L_entr \\
& Fp1, Fp2, T7, O1, O2 & \\
AE vs. ASD & Central and occipital & L_max \\
& Fz, F4, C3 CZ, C4, P3, Pz, P4, O1, O2 & No significant differences \\
Control 1 vs. Control 2 & No significant differences & \\
\hline
\end{tabular}


Table 4 L_max group values and significant group differences are shown

\begin{tabular}{|c|c|c|c|c|c|}
\hline \multirow{2}{*}{ Region } & \multirow{2}{*}{$\begin{array}{l}\text { Sensor } \\
\text { location }\end{array}$} & \multirow{2}{*}{$\begin{array}{l}\mathrm{p} \text {-value for } \\
\text { abs-ASD } \\
\text { difference } \\
\text { for } \mathrm{p}<10^{-4}\end{array}$} & \multicolumn{3}{|c|}{ L_max value } \\
\hline & & & Abs & ASD & Con \\
\hline \multirow{6}{*}{ Frontal } & Fp1 & & 234 & 278 & 715 \\
\hline & Fp2 & & 227 & 274 & 648 \\
\hline & F7 & & 285 & 419 & 409 \\
\hline & F3 & & 431 & 576 & 642 \\
\hline & F4 & & 402 & 577 & 636 \\
\hline & F8 & & 288 & 433 & 574 \\
\hline \multirow{5}{*}{ Central } & $\mathrm{Fz}$ & $10^{-5}$ & 521 & 693 & 687 \\
\hline & $\mathrm{Cz}$ & $10^{-5}$ & 520 & 672 & 632 \\
\hline & $\mathrm{Pz}$ & $10^{-5}$ & 458 & 613 & 643 \\
\hline & $\mathrm{C} 3$ & $10^{-4}$ & 491 & 652 & 577 \\
\hline & $\mathrm{C} 4$ & $10^{-5}$ & 460 & 645 & 609 \\
\hline \multirow{2}{*}{ Temporal } & $\mathrm{T} 7$ & & 309 & 458 & 716 \\
\hline & T8 & & 297 & 420 & 406 \\
\hline \multirow{4}{*}{ Parietal } & P7 & & 356 & 495 & 610 \\
\hline & P3 & $10^{-5}$ & 438 & 611 & 635 \\
\hline & P4 & $10^{-5}$ & 431 & 605 & 466 \\
\hline & P8 & & 364 & 463 & 661 \\
\hline \multirow{2}{*}{ Occipital } & $\mathrm{O} 1$ & $10^{-5}$ & 324 & 495 & 633 \\
\hline & $\mathrm{O} 2$ & & 327 & 479 & 658 \\
\hline
\end{tabular}

Yellow shading indicates locations where absence and ASD groups differ significantly. While the absence values are much lower than controls almost everywhere, ASD deviations from controls are primarily limited to Fp1,2 and O1,2 regions

\section{Absence versus ASD}

Although the absence and ASD groups differed from controls in similar locations, when compared to each other the only significant group differences were found in the L_max features in central locations. Importantly, L_max values for absence cases in Central (Fz, Cz, Pz, C3, C4) and Parietal regions (P3, P4, and O1) were significantly lower than for the ASD cases. However, control and ASD values in these regions were similar.

For all group comparisons, and for the feature ranking calculations discussed below, L_max is the most useful feature for differentiating the absence, ASD and control groups. Thus, group mean multiscale values for L_max are shown in Table 4 for each sensor location and each group. The yellow highlighted values are those that differ significantly between absence and ASD groups. L_max is lowest in the absence group at every location. L_max values for the ASD group are intermediate between absence and controls at most locations, though higher than the controls at F7, Fz, Cz, C3, C4, T8, and P4. With the exception of T8, these are also the locations where absence and ASD groups differ most significantly. 


\section{Machine learning classification}

The complexity index for each nonlinear measure, and each scalp location, was used as input to machine learning classification algorithms. The result of these classification calculations is shown in Table 5. The AE versus control classification was 100\%, and ASD versus controls was $97 \%$ (a single false positive error). AE versus ASD classification was less accurate (75, 72 sensitivity for $\mathrm{AE}$ and $77 \%$ specificity), suggesting that the $\mathrm{AE}$ and ASD subjects were much harder to differentiate from each other than from the controls. The empirical p-values shown were computed using the permutation tests method described in the methods section [54]. Classification results are shown in Table 5.

Although it would seem that the highest ranked features would be those that are statistically the most different in two groups, for classification this may not be the case. The reason is that a variable such as TT, though not statistically different in any pair of groups, may introduce an added, independent dimension to another variable such as L_max. Together they may distinguish group members more clearly than either alone. The highest ranked features for classification are those that are found to contribute the most information to the classifier.

In summary, the absence and ASD groups can be classified from either or both control groups with nearly perfect accuracy. Although there is some overlap between the absence and ASD groups, the classification accuracy of $75 \%$ is significantly better than chance, as demonstrated by the permutation tests used to compute the empirical $p$-values, as explained earlier in the methods section. The classification results are illustrated in Fig. 3, which also suggests a decision support application for screening in a clinical setting.

Figures 4, 5, 6, 7 show multiscale curves for each of the three groups (AE, ASD, controls) at each scalp location, for each RQA or muliscale entropy value. Shading was used to show where the most significant differences were between each pair of groups: $\mathrm{AE}$ versus controls (red shading) and ASD versus controls (green shading). The features that exhibit the greatest differences are L_max, the two entropy measures (SampE and L_entr) and TT. Figure 8 shows scalp plots with multiscale graphs of L_max and SampE for AE versus ASD groups. These differ primarily in central regions of the scalp where the AE cases differ significantly from controls, but ASD cases do not. L_max values for AE cases in Central (Fz, Cz, Pz, C3, C4) and Parietal regions (P3, $\mathrm{P} 4$, and $\mathrm{O} 1$ ) were significantly lower than for the autism cases. One interpretation of this is that atypical AE features are more widespread across the scalp than in ASD.

The AE group differed significantly from the controls at most sensor locations across the scalp for one or more signal features when comparing the mean value of the curve across all scales. EEG segments were chosen from AE cases from inter-ictal periods

Table 5 Classification results for the decision tree shown in Fig. 3

\begin{tabular}{|c|c|c|c|c|c|}
\hline \multirow[t]{2}{*}{ Groups } & \multicolumn{3}{|c|}{ Classification } & \multirow{2}{*}{$\begin{array}{l}\text { Empirical } \\
p \text {-value }\end{array}$} & \multirow{2}{*}{$\begin{array}{l}\text { Highest } \\
\text { ranked } \\
\text { features }\end{array}$} \\
\hline & Accuracy & Sensitivity & Specificity & & \\
\hline AE versus controls & 1.00 & 1.00 & 1.00 & $<0.01$ & L_max, TT \\
\hline ASD versus controls & 0.97 & 1.00 & 0.94 & $<0.01$ & L_max, $T$ \\
\hline AE versus ASD & 0.75 & 0.72 & 0.77 & $<0.01$ & L_max \\
\hline Control 1 versus Control 2 & \multicolumn{5}{|c|}{ Control groups cannot be distinguished. } \\
\hline
\end{tabular}

Empirical $p$-values are computed using the method of shuffled labels as described in [38] 


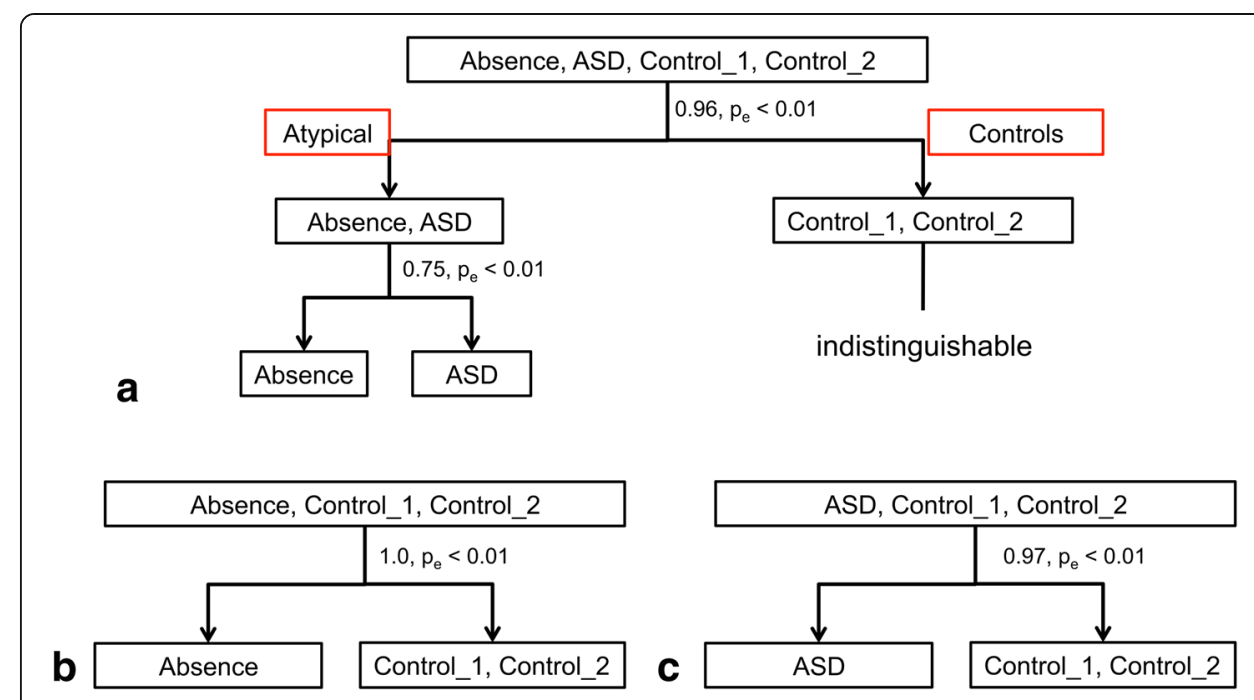

Fig. 3 Decision tree showing accuracy of classification for absence, autism, and control groups. a Controls versus disease cases and absence versus autism; $\mathbf{b}$ absence versus controls; $\mathbf{c}$ autism versus controls

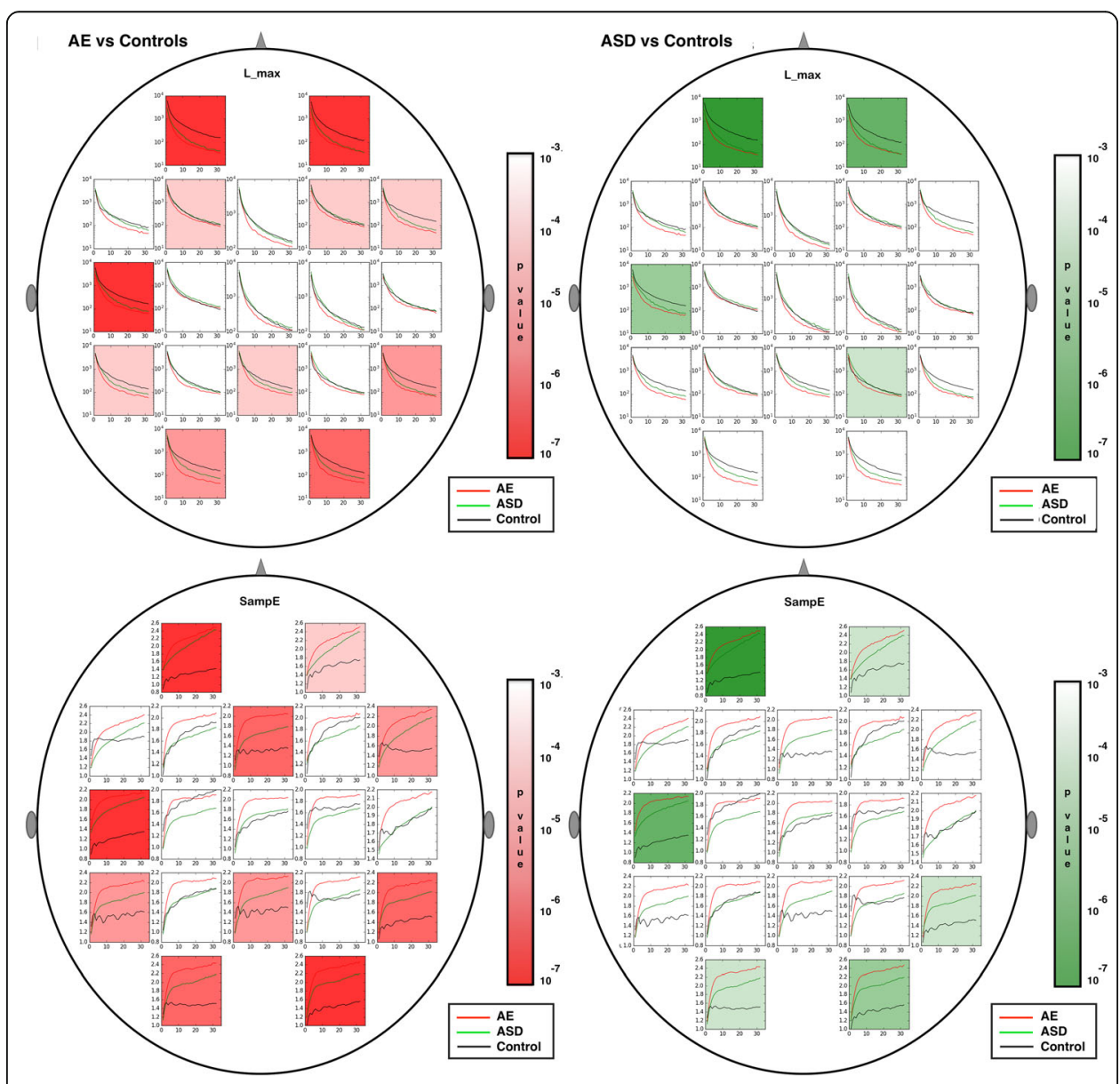

Fig. 4 Scalp plots for multiscale RQA measures are shown for absence epilepsy (AE), autism (ASD) and controls. Regions where significant differences existed $(p<.0001)$ are shaded in red (AE versus controls) or green (ASD versus controls). From top to bottom: L_max, SampE 


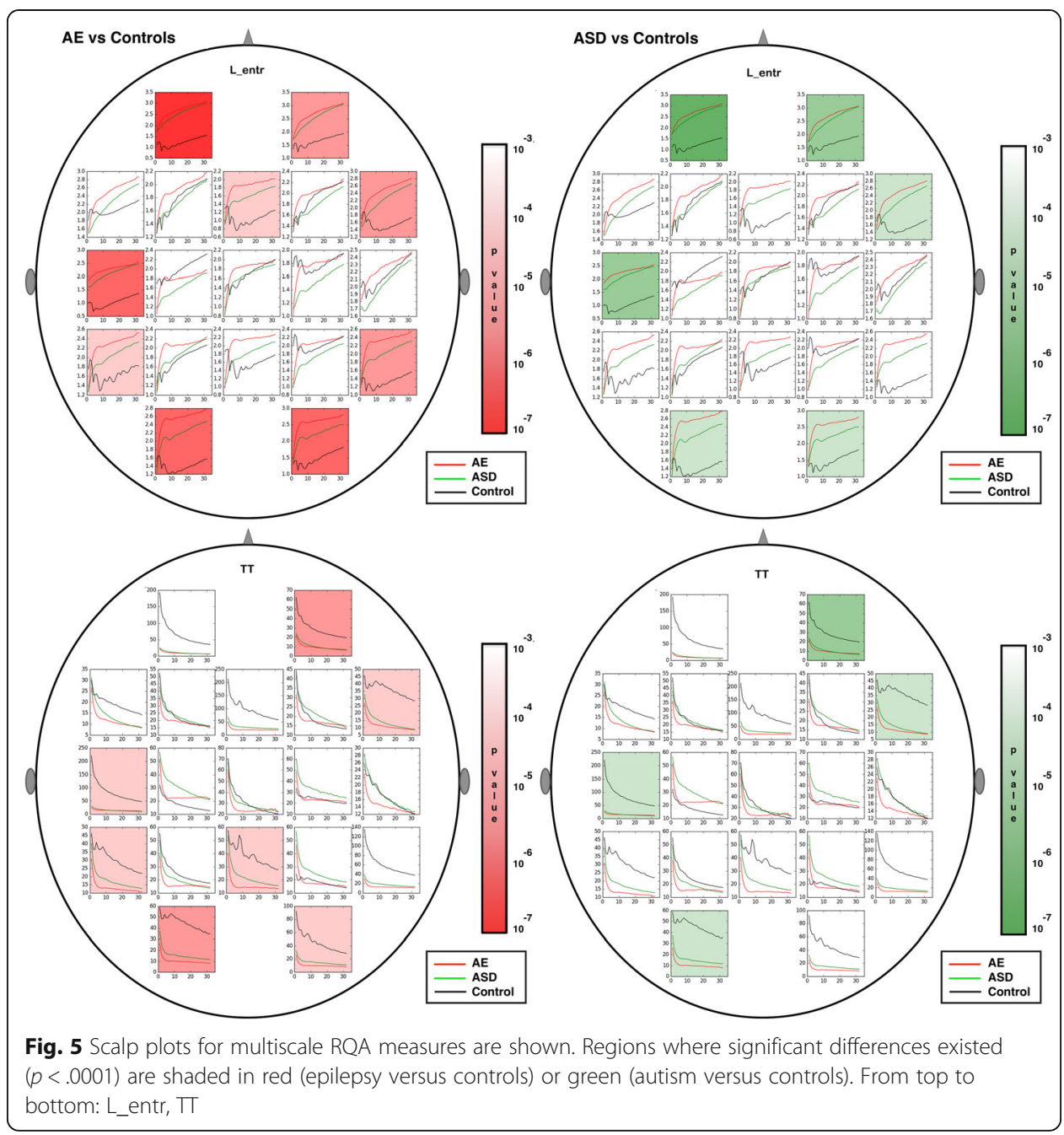

and did not exhibit epileptiform activity in the eye of the neurophysiologist, excluding that visibile interictal epileptiform discharges account for RQA differences. The ASD group also differed significantly from the control groups, but scalp locations were not as widely distributed. The most significant differences were in orbitofrontal (Fp1, Fp2), left temporal (T7) and occipital $(\mathrm{O} 1, \mathrm{O} 2)$ regions. Absence and ASD groups were more difficult to differentiate from each other than from controls.

For all group comparisons, and for the feature ranking calculations discussed below, L_max is the most useful feature for differentiating the absence, ASD and control groups. Thus, group mean multiscale values for L_max are shown in Table 4 for each sensor location and each group. The yellow highlighted values are those that differ significantly between absence and ASD groups. L_max is lowest in the absence group at every scalp location. L_max values for the ASD group are intermediate between absence and controls at most locations, and indistinguishable from controls in central regions. Multiscale L_max and sample entropy curves are shown in Fig. 8. Gray shading is used to indicate where significant differences between mean values between epilepsy and ASD cases are found. 


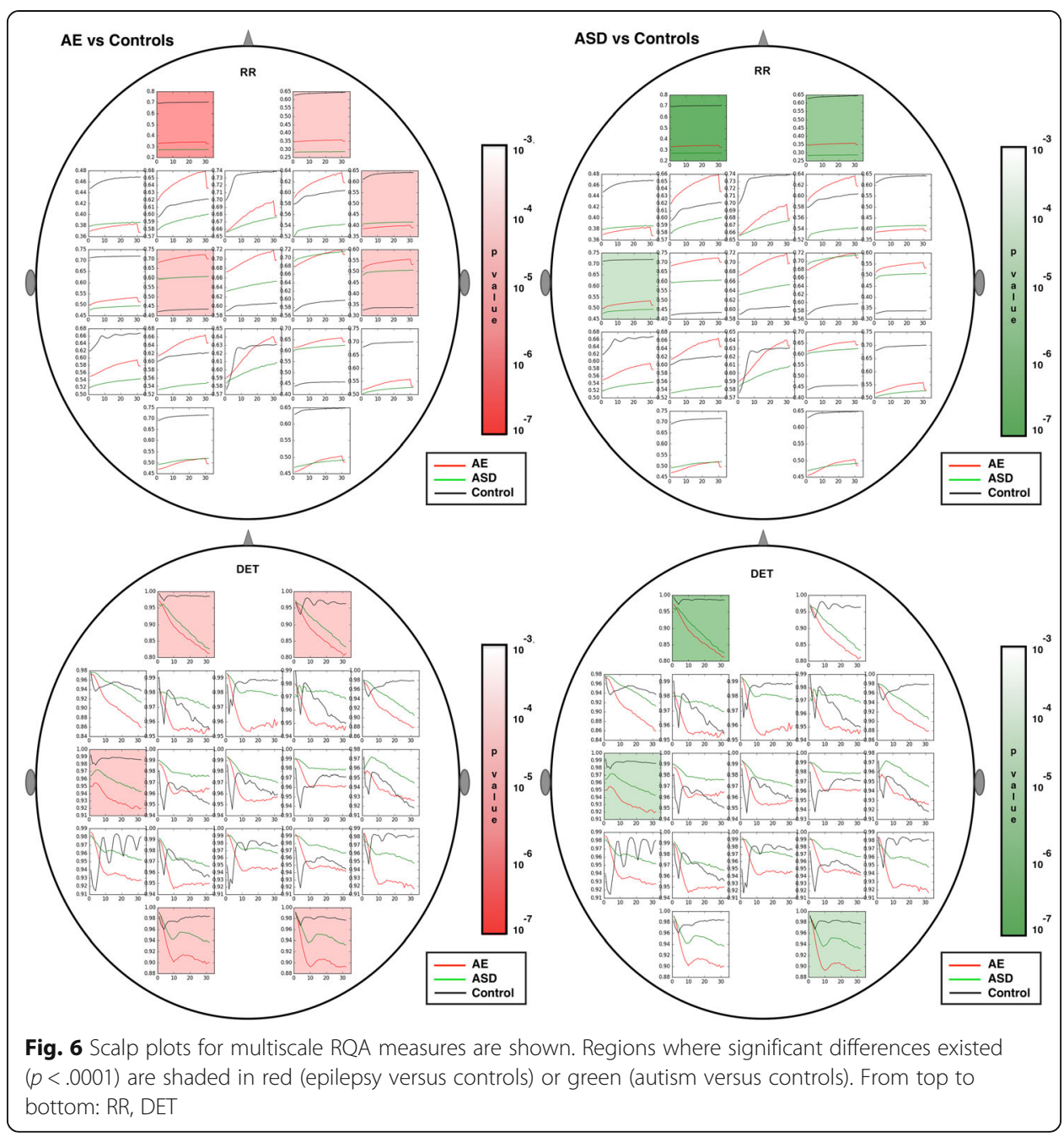

\section{Discussion}

Three general findings resulted that suggest RQA analysis may be useful for neurophysiological research and future clinical applications. First, we found highly significant differences in the multiscale RQA curves between absence, ASD and control groups. Machine learning classifiers were able to distinguish AE cases from controls with $100 \%$ accuracy and ASD groups from controls with an accuracy of $97 \%$ (100\% sensitivity, $94 \%$ specificity), as shown in Table 5. P-values shown were derived by a permutation test procedure that involves randomly shuffling group labels and performing the classification with the random labels [54]. Secondly, the two control groups were indistinguishable. Not only were there no significant control group differences in any of the values or scalp locations, but also machine learning algorithms were unable to distinguish the two control groups using any combination of the RQA features ( $p>0.9$ using the permutation procedure of [54]) This is an important finding, because learning algorithms can find differences that may be due to factors unrelated to those of interest, such as equipment differences. Finally, the most significant spatial locations that differentiated the absence and ASD groups from controls and from each other may give some insight into the neuronal dynamics that characterize these conditions. We discuss these findings in more depth below. 


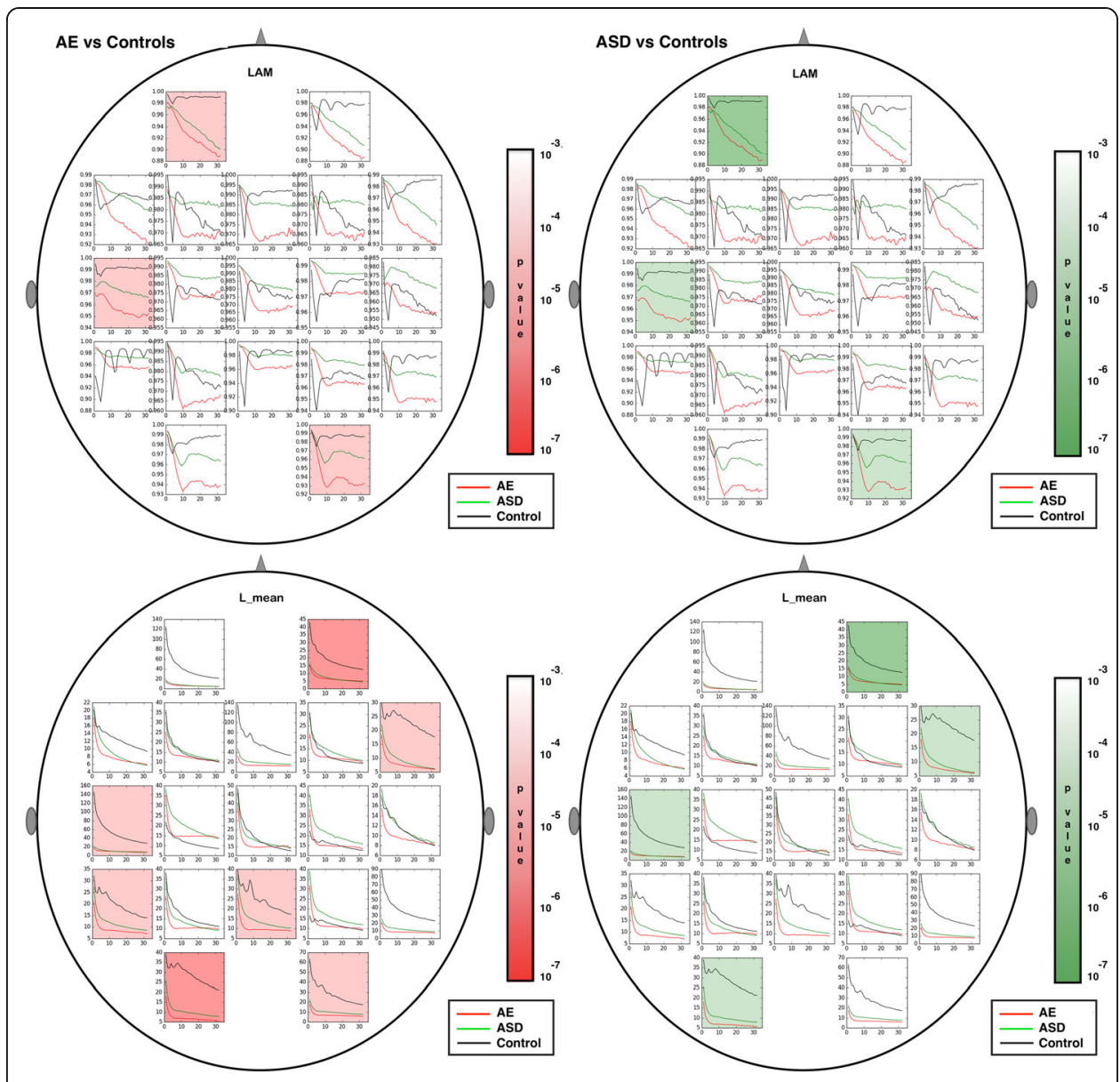

Fig. 7 Scalp plots for multiscale RQA measures are shown. Regions where significant differences existed $(p<.0001)$ are shaded in red (epilepsy versus controls) or green (autism versus controls). From top to bottom: LAM, L_mean

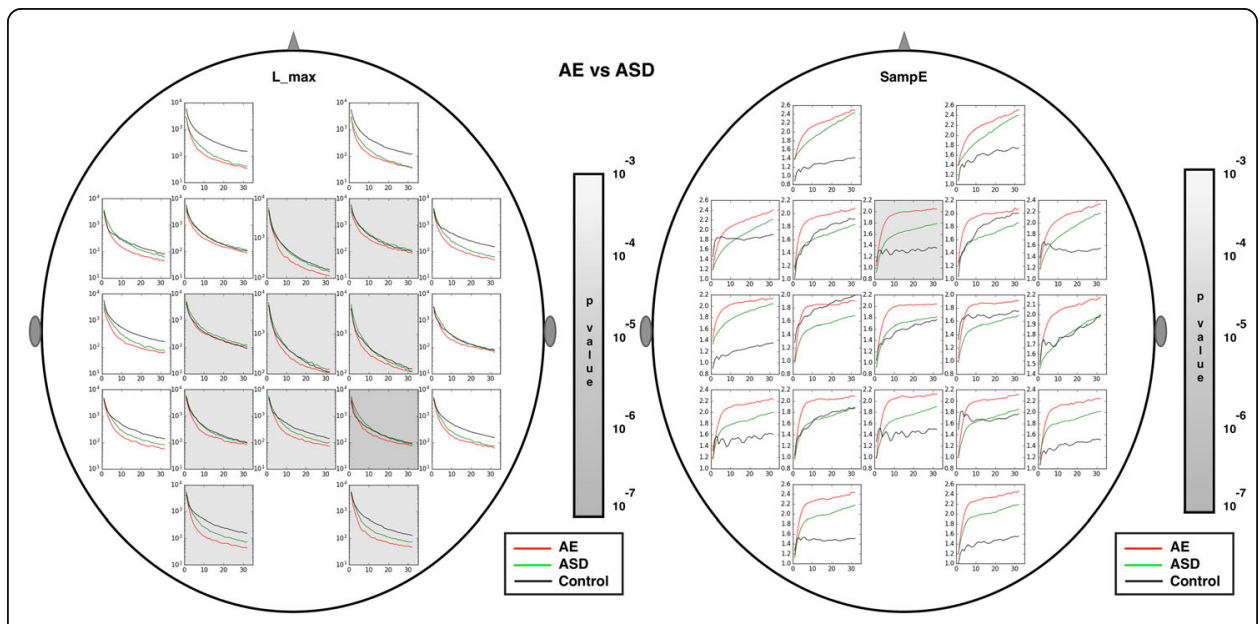

Fig. 8 Significant differences between epilepsy and autism cases are shaded in gray. Only two dynamical variables, L_max and SampE, revealed significant differences between autism and epilepsy. left: L_max, right: SampE 


\section{Control groups}

Our initial expectation was that the control groups would be significantly different from each other on at least some measures, since we thought that differences in laboratory versus clinical settings and the different EEG equipment would introduce systematic differences in the multiscale RQA values. This was not the case. The two control groups had statistically indistinguishable complexity indices for entropy and RQA values at every sensor location and for every dynamical variable. Furthermore, in a 10 -fold cross validation scheme, machine learning classifiers were unable to differentiate the two control groups with accuracy better than random chance. This was demonstrated using a permutation procedure to compute empirical $p$-values [54].

This was in stark contrast to the nearly perfect classification accuracy when either absence or ASD groups were classified with either or both control groups.

The controls from the ASD research study (Laboratories for Cognitive Neuroscience) were carefully screened to not have any neurological or mental disorders. The controls selected from the $\mathrm{BCH}$ Epilepsy Center were not so carefully screened, as might be expected from a real clinical setting. Although these controls were determined to not have epilepsy, and none were known to have ASD, they nevertheless were examined in a tertiary neurology unit for other problems that ultimately were determined to include conditions such as daydreaming, syncope, night terrors or sleepwalking. It is thus surprising that no differences were found between the two control groups.

\section{Detection of AE and ASD}

Cognitive processes are the result of transient synchronization of local and distributed neuronal assemblies [57]. Neuronal oscillations must balance the need for transient synchronization and the pathological, runaway hyper-synchronization that results in an epileptic seizure. The Lyapunov exponent is a measure of the amount of 'chaos' a time series exhibits. The L_max value computed in this study is related to the largest Lyapunov exponent of a time series [36]. The more chaotic a signal is, the higher the Lyapunov exponent is, the more quickly it diverges from an initial trajectory when slightly perturbed. Because of their greater propensity to diverge from an initial starting point, chaotic signals can entrain and synchronize only transiently before diverging from each other. Lower chaos is often an indication of an unhealthy physiological condition [51].

AE subjects revealed significantly different values at nearly all scalp locations in some of the RQA values, and particularly L_max. Smaller L_max indicates less chaotic signals that may synchronize for longer periods, increasing the probability of hypersynchronization over larger neuronal assemblies. Thus, lower L_max is consistent with the AE group. As shown in Table 3, the absence group has lower L_max values than the controls at every sensor location. The ASD group is mixed: at some locations it has a similar L_max value as controls. At several key locations (Fp1, Fp2, T7, P8, O1, O2) the L_max values of the ASD group are significantly lower than controls, but higher than in the absence cases.

The absence subjects have much larger average trapping time (TT) values, though the variability of TT values is greater, hence group differences are less significant. Larger TT values indicate that the time series remain in a similar state for longer periods (the time they are 'trapped' in a state is longer), which is consistent with lower chaotic activity. This 
might be interpreted as creating a greater probability of massive entrainment and hypersynchronization.

\section{Scalp location of most significant features}

The ASD group differed significantly from controls for most features at Fp1, Fp2, T7, P8, O1, O2, as noted previously. At these locations, the L_max values for ASD were midway between the absence and control values, as for many other features (see Table 3). This finding suggests that a common pathology may be involved that results in lesser or different symptom manifestations in ASD when compared to AE cases.

The absence and ASD groups differed from each other primarily in central regions. One interpretation of this is that absence features differ from controls in most scalp regions whereas ASD atypicalities are focused in orbitofrontal, left temporal and occipital regions. At most locations the ASD values were midway between the absence and control values. Since seizures may involve a synchronization process that entrains multiple frequency bands leading to large amplitude, highly periodic and low complexity activity, we speculate that the propensity to have seizures is represented by multiresolution dynamics. That is, the epileptic brain differs from non-epileptic brain over many time or frequency scales. Summing derived values over many scales, such as with the complexity index, includes some information from every scale. In biological systems, a lower level of chaotic dynamics is often associated with pathological conditions [58-62].

Classification results shown in Table 4 reveal two important points. First, both AE and ASD groups can accurately be distinguished from the controls. The AE and ASD groups cannot be classified quite as well, with an accuracy of $75 \%$, perhaps suggesting a common pathology and fundamental similarities in neural network structures, at least in some locations as discussed previously. Or, it may be that RQA analysis only identifies a coarse-grained distinction between typical and atypical subjects, and is less effective at distinguishing specific neuropathology.

We note again that the significance of group differences is computed from the mean values derived from the multiscale curves. Future studies may take into account differences across the entire multiscale curves, such as slope or area under the curve. Machine learning classifiers might also use more information from the multiscale curves if an appropriate metric is defined for differences between curves.

The above results reveal three important conclusions. First, the AE and ASD groups each differ significantly from the control groups in many sensor locations and for 5 of the 8 signal features. L_max is the most useful biomarker for distinguishing these groups, although other RQA values may also contribute useful information to bioprofiles for $\mathrm{AE}$ and ASD. Second, AE and ASD groups differ significantly from each other primarily in centrally located regions on the L_max feature. And finally, the two control groups are indistinguishable on the basis of the signal features discussed in this paper, even though they were measured in different settings using different EEG equipment, suggesting that the features contain information that is independent of lab and EEG equipment.

\section{Confounding factors and further study}

Data and controls need to be interpreted in the clinical setting from which data was acquired. Several confounding factors require further study. The control subjects from 
the $\mathrm{BCH}$ Epilepsy Clinic used in this study may have a variety of neurological disturbances that have not fully emerged. They presented in the clinic initially because of some neurological concerns, though none was known to have a diagnosis of $\mathrm{AE}$ or ASD. The controls derived from the Laboratories of Cognitive Neuroscience were carefully screened for any known history of neurological or mental disorders. The statistical equivalence of the two control groups found in our analysis gives greater confidence in the appropriateness of both control groups. However, it remains puzzling to us that some differences between the control groups did not appear, since the controls from the Epilepsy Center were evaluated for suspected neurological conditions. The controls from the LCN were carefully screened to not have any known neurological or psychiatric condition.

The gender distribution between the groups differed. Control group 1 was $38 \%$ male, while control group 2 was $91 \%$ male. The statistical similarity of these two groups on nearly all measures suggests that gender was not a significant factor in our analysis. The absence group was 50\% male while the ASD group was $89 \%$ male. The fact that the balance of male/female differed in the two control groups, and the results of our analysis are the same, regardless of which control group was used, also suggests that gender was not a significant factor. Nevertheless, further studies of gender differences with larger study groups may be useful for gaining further insights. Early studies of EEG complexity differences in children at risk for developing ASD based on family history did suggest gender differences [49].

For this initial study, patients with AE were selected because they represent a common diagnosis in the epilepsy population, are relatively easy to characterize clinically and electroencephalographically, and constitute one of the most homogeneous groups of patients with epilepsy. Additionally, there are no clear structural brain abnormalities on structural MRI imaging, and there is no EEG slowing which may confound results in other epilepsies or seizure types. Whether these findings will be applicable to other patients with different types of epilepsy will require additional data sets with patients who have clearly identified epilepsy syndromes. We hypothesize that if RQA analysis is able to detect the dynamics of an epileptic brain, then different epilepsies will reveal different spatial distributions than those seen for absence patients. In particular, focal epilepsies will have atypical RQA values in the epileptic regions.

\section{Conclusions}

Our results demonstrate that EEG complexity, as measured by RQA analysis, may contain information to detect AE, ASD and to distinguish children with these disorders from typically developing children. The signal feature that is most highly ranked, Lmax, is related to the Lyapunov exponent, which may be related to how easily neural oscillators can synchronize, and this is ultimately related to epileptiform or spiking activity and to seizures. Atypical dynamics were found to be more widespread in absence cases than in ASD, but the commonalities may reveal similar neural network pathologies in common scalp locations. Clinically, the classification results found in this patient series suggest that RQA analysis may provide a useful biomarker profile for both epilepsy and ASD, and a quantitative measure for comparing similarities and differences between these common pediatric conditions. As a next step, larger validation studies are required to confirm and potentially further these biomarkers prospectively. 


\section{Abbreviations}

AE: Absence epilepsy; ASD: Autism spectrum disorder; BCH: Boston children's hospital; EEG: Electroencephalography; fMRI: functional magnetic resonance imaging; LCN: Laboratories for cognitive neuroscience; MEG: Magnetoencephalography; PET: Postitron emission tomography; RQA: Recurrence quantitative analysis; SPECT: Single photon emission computed tomography

\section{Acknowledgments}

We are deeply thankful to Vanessa Farley-Vogel and Geneva Gregorio for their extraordinary ability to work with children in the Laboratories for Cognitive Neuroscience and for patiently preparing data for analysis. We thank Dr. Ivan Sanchez Fernandez for assisting TL in pulling records and reviewing EEG traces from the BCH Epilepsy Center database. We are especially grateful to the families who participated in this study.

\section{Funding}

WB was supported by grants from the National Institute of Mental Health (R21-MH 093753) and the Simons Foundation (137186). CN received support for this research from grants R01-DC010290 and R21 DC 08637 from the National Institutes of Health and Autism Speaks (1323) and Simons Foundation (137186).

\section{Availability of data and materials}

Software for RQA analysis is available at: http://tocsy.pik-potsdam.de/commandline-rp.php. Wavelet transforms and machine learning methods described in this paper are in Python packages scipy and scikit-learn, respectively. Patient data used in this study is not available for research. Subjects from the Laboratories for Cognitive Neuroscience (autism subjects) were not consented for public release of the data and parents could not be re-contacted. Patient data from the $\mathrm{BCH}$ Epilepsy Center could not be properly de-identified for public release.

\section{Authors' contribution}

WB wrote the computer codes for analysis, performed the calculations, and drafted the manuscript. WB, $T L$, and CN jointly designed the study, discussed and contributed to interpretation of the results, and edited the final manuscript. $\mathrm{CN}$ directs and oversees the Laboratories for Cognitive Neuroscience at Boston Children's Hospital and directed the specific project that collected data from the autism subjects in this study and the respective control group. TL is the director of clinical research for the Epilepsy Center at Boston Children's Hospital and pulled absence epilepsy patient and control records at the Epilepsy Center used in this study.

\section{Competing interests}

WJB and TL are named on pending patent applications submitted by the Boston Children's Hospital Technology Development Office that includes parts of the signal analysis methods discussed in this article. TL serves on the Laboratory Accreditation Board for Long Term (Epilepsy and Intensive Care Unit) Monitoring, on the Council (and as $2^{\text {nd }}$ Vice President) of the American Clinical Neurophysiology Society, on the American Board of Clinical Neurophysiology, as an Associate Editor for Seizure, and as an Associate Editor for Wyllie's Treatment of Epilepsy $6^{\text {th }}$ edition. He receives research support from the Epilepsy Research Fund, the American Epilepsy Society, the Epilepsy Foundation of America, the Epilepsy Therapy Project, PCORI, the Pediatric Epilepsy Research Foundation, CURE, HHV-6 Foundation, and received research grants from Lundbeck, Eisai, Upsher-Smith, Acorda, and Pfizer. He serves as a consultant for Zogenix, Upsher Smith and Lundbeck. He performs video electroencephalogram long-term and ICU monitoring, electroencephalograms, and other electrophysiological studies at Boston Children's Hospital and affiliated hospitals and bills for these procedures and he evaluates pediatric neurology patients and bills for clinical care. CAN has no competing financial or nonfinancial interests to disclose.

\section{Consent for publication}

Not applicable.

\section{Ethics approval and consent to participate}

We performed a quantitative retrospective analysis to evaluate the use of nonlinear features derived from RQA analysis of EEG segments as biomarkers for absence epilepsy and autism spectrum disorders. The Institutional Review Board at Boston Children's Hospital granted approval for this study. Given its retrospective nature, the need to obtain individual informed consents was waived. Written informed assent was obtained from each participant and one of their parents, and verbal assent was confirmed from each participant prior to the experiment for all participants in the autism research at the Laboratories of Cognitive Neuroscience at Boston Children's Hospital. The epilepsy cases were obtained retrospectively from a review of the records in the Epilepsy Center at Boston Children's Hospital, thus the need to obtain individual informed consents was waived by the Institutional Review Board at Boston Children's Hospital.

\section{Publisher's Note}

Springer Nature remains neutral with regard to jurisdictional claims in published maps and institutional affiliations.

\section{Author details}

${ }^{1}$ Program in Health Informatics and Clinical Psychology, University of San Francisco, San Francisco, CA, USA. ${ }^{2}$ Benioff Children's Hospital Oakland Research Institute, Oakland, CA, USA. ${ }^{3}$ Department of Neurology, Boston Children's Hospital, Boston, MA, USA. ${ }^{4}$ Laboratories of Cognitive Neuroscience, Division of Developmental Medicine, Boston Children's Hospital, Boston, MA, USA. ${ }^{5}$ Harvard Medical School, Boston, MA, USA. 
Received: 1 November 2016 Accepted: 8 March 2017

Published online: 23 March 2017

\section{References}

1. Tuchman R, Hirtz D, Mamounas LA. NINDS epilepsy and autism spectrum disorders workshop report. Neurology. 2013;81(18):1630-6. doi:10.1212/WNL.0b013e3182a9f482. Epub 2013/10/04.

2. Tuchman R, Alessandri M, Cuccaro M. Autism spectrum disorders and epilepsy: moving towards a comprehensive approach to treatment. Brain Dev. 2010;32(9):719-30. doi:10.1016/j.braindev.2010.05.007. Epub 2010/06/19.

3. Besag FM. The relationship between epilepsy and autism: a continuing debate. Acta Paediatr. 2009;98(4):618-20 doi:10.1111/j.1651-2227.2008.01190.x. Epub 2009/02/13.

4. Spence SJ, Schneider MT. The role of epilepsy and epileptiform EEGs in autism spectrum disorders. Pediatr Res. 2009;65(6):599-606. doi:10.1203/PDR.0b013e31819e7168. PubMed PMID: 19454962; PubMed Central PMCID: PMC2692092.

5. Berg AT, Plioplys S, Tuchman R. Risk and correlates of autism spectrum disorder in children with epilepsy: a community-based study. J Child Neurol. 2011;26(5):540-7. doi:10.1177/0883073810384869. PubMed PMID: 21421903; PubMed Central PMCID: PMC3085568.

6. Gilby KL, O'Brien TJ. Epilepsy, autism, and neurodevelopment: kindling a shared vulnerability? Epilepsy Behav. 2013;26. doi:10.1016/j.yebeh.2012.11.002.

7. El Achkar CM, Spence SJ. Clinical characteristics of children and young adults with co-occurring autism spectrum disorder and epilepsy. Epilepsy Behav. 2015;47:183-90. doi:10.1016/j.yebeh.2014.12.022.

8. Ben-Shalom R, Keeshen CM, Berrios KN, An JY, Sanders SJ, Bender KJ. Opposing effects on NaV1.2 function underlie differences between SCN2A variants observed in individuals with autism spectrum disorder or infantile seizuresSCN2A/NaV1.2 mutations underlying autism and seizure. Biol Psychiatry. 2017;81(6). doi:10.1016/ j.biopsych.2017.01.009.

9. Harstad EB, Fogler J, Sideridis G, Weas S, Mauras C, Barbaresi WJ. Comparing diagnostic outcomes of autism spectrum disorder using DSM-IV-TR and DSM-5 criteria. J Autism Dev Disord. 2015;45(5):1437-50. doi:10.1007/s10803-014-2306-4.

10. Volkmar FR, MCPartland JC. From Kanner to DSM-5: autism as an evolving diagnostic concept. Annu Rev Clin Psychol. 2014;10:193-212. doi:10.1146/annurev-clinpsy-032813-153710.

11. Baio J. Prevalence of autism spectrum disorder among children aged 8 years - autism and developmental disabilities monitoring network, 11 sites, United States, 2010. MMWR Surveill Summ. 2014;63(2):1-21.

12. Elsabbagh M, Divan G, Koh YJ, Kim YS, Kauchali S, Marcin C, et al. Global prevalence of autism and other pervasive developmental disorders. Autism Res. 2012;5(3):160-79. doi:10.1002/aur.239.

13. Baxter AJ, Brugha TS, Erskine HE, Scheurer RW, Vos T, Scott JG. The epidemiology and global burden of autism spectrum disorders. Psychol Med. 2015;45(3):601-13. doi:10.1017/S003329171400172X

14. Bell GS, Neligan A, Sander JW. An unknown quantity-the worldwide prevalence of epilepsy. Epilepsia. 2014;55(7):958-62. doi:10.1111/epi.12605.

15. Jensen FE. Epilepsy as a spectrum disorder: implications from novel clinical and basic neuroscience. Epilepsia. 2011;52 Suppl 1:1-6. doi:10.1111/j.1528-1167.2010.02904.x. Epub 2011/01/19.

16. Fisher RS, van Emde BW, Blume W, Elger C, Genton P, Lee P, et al. Epileptic seizures and epilepsy: definitions proposed by the International League Against Epilepsy (ILAE) and the International Bureau for Epilepsy (IBE). Epilepsia. 2005;46(4):470-2. doi:10.1111/j.0013-9580.2005.66104.x.

17. Fisher RS, Acevedo C, Arzimanoglou A, Bogacz A, Cross JH, Elger CE, et al. ILAE official report: a practical clinical definition of epilepsy. Epilepsia. 2014;55(4):475-82. doi:10.1111/epi.12550. Epub 2014/04/16.

18. Kobau R, Luo Y, Zack M, Helmers S, Thurman D. Epilepsy in adults and access to care - United States, 2010. MMWR. 2012;61(45):909-13.

19. Deonna T, Roulet E. Autistic spectrum disorder: evaluating a possible contributing or causal role of epilepsy. Epilepsia. 2006;47 Suppl 2:79-82. doi:10.1111/j.1528-1167.2006.00697.x. Epub 2006/11/16.

20. Sanchez Fernandez I, Loddenkemper T, Galanopoulou AS, Moshe SL. Should epileptiform discharges be treated? Epilepsia. 2015;56(10):1492-504. doi:10.1111/epi.13108.

21. Ekinci O, Arman AR, Isik U, Bez Y, Berkem M. EEG abnormalities and epilepsy in autistic spectrum disorders: clinical and familial correlates. Epilepsy Behav. 2010;17(2):178-82. doi:10.1016/j.yebeh.2009.11.014.

22. Tuchman R, Moshe SL, Rapin I. Convulsing toward the pathophysiology of autism. Brain Dev. 2009;31(2):95-103. doi:10.1016/j.braindev.2008.09.009. PubMed PMID: 19006654; PubMed Central PMCID: PMCPMC2734903.

23. Mulligan CK, Trauner DA. Incidence and behavioral correlates of epileptiform abnormalities in autism spectrum disorders. J Autism Dev Disord. 2013;44(2):452-8. doi:10.1007/s10803-013-1888-6. Epub 2013/07/23.

24. Glauser TA, Cnaan A, Shinnar S, Hirtz DG, Dlugos D, Masur D, et al. Ethosuximide, valproic acid, and lamotrigine in childhood absence epilepsy. N Engl J Med. 2010;362(9):790-9. doi:10.1056/NEJMoa0902014. PubMed PMID: 20200383, PubMed Central PMCID: PMC2924476.

25. Dunn DW, Kronenberger WG. Childhood epilepsy, attention problems, and ADHD: review and practical considerations. Semin Pediatr Neurol. 2005;12(4):222-8. doi:10.1016/j.spen.2005.12.004.

26. Duran MH, Guimaraes CA, Montenegro MA, Neri ML, Guerreiro MM. ADHD in idiopathic epilepsy. Arq Neuropsiquiatr. 2014;72(1):12-6. doi:10.1590/0004-282X20130193.

27. Kral MC, Lally MD, Boan AD. Identification of ADHD in youth with epilepsy. J Pediatr Rehabil Med. 2016;9(3):223-9. doi:10.3233/PRM-160383.

28. Li X, Ouyang G, Richards DA. Predictability analysis of absence seizures with permutation entropy. Epilepsy Res. 2007;77(1):70-4. doi:10.1016/j.eplepsyres.2007.08.002. Epub 2007/09/18

29. van Drongelen W, Nayak S, Frim DM, Kohrman MH, Towle VL, Lee HC, et al. Seizure anticipation in pediatric epilepsy: use of Kolmogorov entropy. Pediatr Neurol. 2003;29(3):207-13. Epub 2003/11/25

30. Rosso OA. Entropy changes in brain function. Int J Psychophysiol. 2007;64(1):75-80. doi:10.1016/j.jpsycho.2006.07.010. 
31. Burioka N, Cornelissen G, Maegaki Y, Halberg F, Kaplan DT, Miyata M, et al. Approximate entropy of the electroencephalogram in healthy awake subjects and absence epilepsy patients. Clin EEG Neurosci. 2005;36(3):188-93.

32. Lee HC, van Drongelen W, McGee AB, Frim DM, Kohrman MH. Comparison of seizure detection algorithms in continuously monitored pediatric patients. J Clin Neurophysiol. 2007;24(2):137-46. doi:10.1097MNP.0b013e318033715b. Epub 2007/04/07.

33. Catarino A, Churches $\mathrm{O}$, Baron-Cohen S, Andrade A, Ring H. Atypical EEG complexity in autism spectrum conditions: A multiscale entropy analysis. Clin Neurophysiol. 2011;122(12):2375-83.

34. Bosl W, Tierney A, Tager-Flusberg H, Nelson C. EEG complexity as a biomarker for autism spectrum disorder risk. BMC Med. 2011;9:18.

35. Eldridge J, Lane AE, Belkin M, Dennis $\mathrm{S}$. Robust features for the automatic identification of autism spectrum disorder in children. J Neurodevs Dis. 2014;12:6.

36. Marwan N, Romano MC, Thiel M, Kurths J. Recurrence plots for the analysis of complex systems. Phys Rep 2007:438:237-329.

37. Schinkel S, Marwan N, Kurths J. Brain signal analysis based on recurrences. J Physiol Paris. 2009;103(6):315-23. doi:10.1016/j.jphysparis.2009.05.007. Epub 2009/06/09.

38. Zbilut JP, Thomasson N, Webber CL. Recurrence quantification analysis as a tool for nonlinear exploration of nonstationary cardiac signals. Med Eng Phys. 2002;24(1):53-60. Epub 2002/03/14.

39. Webber $\mathrm{CL}$, Zbilut JP. Recurrence quantitative analysis of nonlinear dynamical systems. In: Riley MA, Van Orden GC, editors. Tutorials in contemporary nonlinear methods for the behavioral sciences. p. 26-94. http://www.nsf.gov/sbe/ bcs/pac/nmbs/nmbs.jsp. Retrieved 1 Mar 2005.

40. Komalapriya C, Thiel M, Romano MC, Marwan N, Schwarz U, Kurths J. Reconstruction of a system's dynamics from short trajectories. Phys Rev E Stat Nonlin Soft Matter Phys. 2008;78(6 Pt 2):066217. Epub 2009/03/05.

41. Webber CL, Marwan N, editors. Recurrence quantification analysis. New York: Springer; 2015.

42. Acharya UR, Sree SV, Chattopadhyay S, Yu W, Ang PC. Application of recurrence quantification analysis for the automated identification of epileptic EEG signals. Int J Neural Syst. 2011;21(3):199-211. doi:10.1142/S0129065711002808.

43. Chen L, Zou J and Zhang J. Dynamic feature extraction of epileptic EEG using recurrence quantification analysis, Beijing: Proceedings of the 10th World Congress on Intelligent Control and Automation; 2012. pp. 5019-5022. doi:10.1109/WCICA.2012.6359429.

44. Niknazar M, Mousavi SR, Vahdat BV, Sayyah M. A new framework based on recurrence quantification analysis for epileptic seizure detection. IEEE J Biomed Health Inform. 2013;17(3):572-8.

45. Heunis TM, Aldrich C, de Vries PJ. Recent advances in resting-state electroencephalography biomarkers for autism spectrum disorder-a review of methodological and clinical challenges. Pediatr Neurol. 2016;61:28-37. doi:10.1016/j.pediatrneurol.2016.03.010.

46. Jasper HH. Report of the committee on methods of clinical examination in electroencephalography: 1957. Electroencephalogr Clin Neurophysiol. 1958;10(2):370-5. doi:10.1016/0013-4694(58)90053-1.

47. Walker JS. A primer on wavelets and their scientific applications. 2nd ed. Boca Raton: Chapman \& Hall/CRC; 2008. p. 300.

48. Marwan N. Commandline Recurrence Plots, Version: 1.13z (Last mod: 2006-03-08). http://tocsy.pik-potsdam.de/ commandline-rp.php. Accessed 28 Sept 2009.

49. BosI W, Tierney A, Tager-Flusberg H, Nelson C. EEG complexity as a biomarker for autism spectrum disorder risk. BMC Medicine. 2011;9:18. doi:10.1186/1741-7015-9-18.

50. Weng WC, Jiang GJ, Chang CF, Lu WY, Lin CY, Lee WT, et al. Complexity of multi-channel electroencephalogram signal analysis in childhood absence epilepsy. PLoS One. 2015;10(8):e0134083. doi:10.1371/journal.pone.0134083. PubMed PMID: 26244497; PubMed Central PMCID: PMCPMC4526647.

51. Costa M, Goldberger AL, Peng CK. Multiscale entropy analysis of biological signals. Phys Rev E Stat Nonlin Soft Matter Phys. 2005;71(2 Pt 1):021906.

52. Fournier KA, Amano S, Radonovich KJ, Bleser TM, Hass CJ. Decreased dynamical complexity during quiet stance in children with autism spectrum disorders. Gait Posture. 2014;39(1):420-3. doi:10.1016/j.gaitpost.2013.08.016.

53. James G, Witten D, Hastie T, Tibshirani R. An introduction to statistical learning: with applications in R, vol. xvi. New York: Springer; 2013. p. 426.

54. Golland P, Fischl B. Permutation tests for classification: towards statistical significance in image-based studies. Inf Process Med Imaging. 2003;18:330-41. Epub 2004/09/04.

55. Lu WY, Chen JY, Chang CF, Weng WC, Lee WT, Shieh JS. Multiscale entropy of electroencephalogram as a potential predictor for the prognosis of neonatal seizures. PLoS One. 2015;10(12):e0144732. doi:10.1371/journal. pone.0144732. PubMed PMID: 26658680; PubMed Central PMCID: PMCPMC4676749.

56. Ngamga EJ, Bialonski S, Marwan N, Kurths J, Geier C, Lehnertz K. Evaluation of selected recurrence measures in discriminating pre-ictal and inter-ictal periods from epileptic EEG data. Phys Lett A. 2016;380(16):1419-25. doi:10.1016/J.PHYSLETA.2016.02.024

57. Rapp PE, Keyser DO, Albano A, Hernandez R, Gibson DB, Zambon RA, et al. Traumatic brain injury detection using electrophysiological methods. Front Hum Neurosci. 2015;9:11. doi:10.3389/fnhum.2015.00011. PubMed PMID: 25698950, PubMed Central PMCID: PMC4316720.

58. Catarino A, Churches O, Baron-Cohen S, Andrade A, Ring H. Atypical EEG complexity in autism spectrum conditions: a multiscale entropy analysis. Clin Neurophysiol. 2011;122(12):2375-83. doi:10.1016/j.clinph.2011.05.004.

59. Costa M, Goldberger AL, Peng CK. Broken asymmetry of the human heartbeat: loss of time irreversibility in aging and disease. Phys Rev Lett. 2005;95(19):198102.

60. Goldberger AL. Fractal variability versus pathologic periodicity: complexity loss and stereotypy in disease. Perspect Biol Med. 1997:40(4):543-61. Epub 1997/07/01.

61. Takahashi T, Cho RY, Mizuno T, Kikuchi M, Murata T, Takahashi K, et al. Antipsychotics reverse abnormal EEG complexity in drug-naive schizophrenia: a multiscale entropy analysis. Neuroimage. 2010;51(1):173-82. doi:10.1016/j.neuroimage.2010.02.009. Epub 2010/02/13.

62. Zhang D, Ding H, Liu Y, Zhou C, Ye D. Neurodevelopment in newborns: a sample entropy analysis of electroencephalogram. Physiol Meas. 2009;30(5):491-504. 